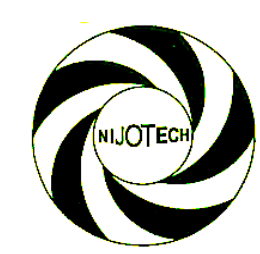

Nigerian Journal of Technology (NIJOTECH)

Vol. 38, No. 2, April 2019, pp. $\mathbf{4 2 2} \mathbf{- 4 3 6}$

Copyright@ Faculty of Engineering, University of Nigeria, Nsukka,

Print ISSN: 0331-8443, Electronic ISSN: 2467-8821 www.nijotech.com

http://dx.doi.org/10.4314/njt.v38i2.19

\title{
MEASURING MOBILE BROADBAND PERFORMANCE IN NIGERIA: 2G AND 3G
}

\author{
F. M. Dahunsi ${ }^{1, *}$ and A. A. Akinlabi ${ }^{2}$ \\ 1,2, Electrical And Electronics Engineering Department, The Federal University of TeChnOlogy, \\ AKURE, ONDO STATE, NIGERIA \\ E-mail addresses: ${ }^{1}$ fmdahunsi@futa.edu.ng, ${ }^{2}$ oluwanipinmi4994@gmail.com
}

\begin{abstract}
With increase in broadband penetration rate in Nigeria there is very little known customer-centric mobile broadband performance analysis in the country, despite the inherent advantages associated with performance monitoring to regulators, operators, content-developers, and most especially the customers. There exists an information gap and customers are curious to know the Quality of Service (QOS) offered them. This paper presents a host and crowdsourced based approach to mobile broadband performance metric measurement and evaluation. A mobile broadband performance measurement application (MBPerf) was developed using Java and Extensible Markup Language (XML) and installed on volunteers' Android Smartphones to measure and collect data relating to 4 (four) QoS metrics - download and upload speeds, latency and DNS (Domain Name Service) lookup; and user data such as mobile phone information, network information, and location information. Measurements were taken for a period of 3 months within Akure and Ibadan metropolis from the 4 major MNOS' (MNO-A, MNO-B, MNO-C and MNO-D) networks in Nigeria. Data was retrieved from the cloud, pre-processed, sorted and analysed using Microsoft Excel version 13 and SPSS (Statistical Package for the Social Sciences) Statistics 19. Findings reveal that 36 users are not getting the industry set speeds. They get about $10 \%$ below the lower limit of the benchmark (500 kilobits per second). However, $2 G$ users get a better deal of about $61 \%$ above the lower limit of the benchmark (100 kilobits per second). It was inferred that network performance is highly unpredictable and variable during the day (between 8 am and $5 \mathrm{pm}$ ) but greatly improves at the early hours of the morning (between 12am to 6am) with a difference of about $69 \%$ between the peak and worst performance. The study indicates that performance deteriorates at peak times (between 7 pm and $11 \mathrm{pm})$. Lastly the DNS performance analysis suggests that the MNOs' DNS servers operate effectively and do not add significant delay to end users' queries.
\end{abstract}

Keywords: Mobile broadband performance, Quality of Service (QoS), crowdsourcing, MBPerf application, hostbased

\section{INTRODUCTION}

Broadband provides high speed, reliable, cost effective and consistent connection to the Internet. Before the advent of broadband, accessibility to the Internet was mainly through dial up access which was limited to $56 \mathrm{Kbps}$ unlike broadband which has traditional capacity of $256 \mathrm{Kbps}$. Broadband uses various mediums to transfer data, such as Digital Subscriber Line (DSL), cable modem, Fiber, wireless and Satellite [1]. Mobile broadband is a high speed connection to the internet using mobile devices such as mobile phones.

Today, the Internet drives a large portion of daily life activities. It has in fact become an integral part of everyday tasks, relating to health, education, business, entertainment, social life and news. Thus, networks now, more than ever, need to operate dynamically in a diverse range of scenarios and still assure a good service quality and user experience [2]. Nigeria's teledensity grew from $16.27 \%$ in 2010 to $120.79 \%$ for the year 2018; the broadband and

\footnotetext{
* Corresponding author, tel: $+234-810-156-4160$
} 
Internet penetration rates stands at $30.9 \%$ and $56.8 \%$ respectively by 2018 [3]. The Ericsson Consumer Lab country report of 2015 also revealed that $82 \%$ of customers in Nigeria access broadband through their mobile Smartphones [4]. The change in focus by many operators from voice to data are some of the stunning reports and statistics that proves that activities in the broadband space of Nigeria are on the rise and are sure to increase even more the broadband penetration rate, particularly on mobile devices as forecasted in the National Broadband Plan - 2013 - 2018 [5].

However, despite these remarkable progress made, there exist an information gap between mobile customers and MNOs in the country on service performance as customers are always curious to know the level of service offered to them by their respective operators. Searching through literature, there is no systematic approach to mobile broadband performance monitoring, analysis and reporting in the country especially customer-based study of mobile broadband performance. A lack of readily available and accessible performance data set is disturbing because aside from cost, performance affects broadband adoption and use, which, in turn, is associated with progress and development [6].

To address the aforementioned, a host and crowdsourced based measurement approach was developed to provide a pool of data, which were analysed to reflect the approximate performance offered to mobile customers in the coverage area of study. Data performance was tested by sending test packets in form of pings to the measurement server, so as to determine the Round Trip Latencies of users' connections; by transferring a payload between the test application (MBPerf) and the measurement server to ascertain the data transfer rates for upload and download speed and by resolving some popular domain names with the help of the MNOs recursive resolvers in order to determine the DNS resolution times. The following research questions were studied:

i. does the speed (upload or download) and latency of each user's mobile broadband connection achieve the benchmark speed for each of the network technologies studied?

ii. what latencies and data transfer rates exist in the coverage area of study?

iii. how responsive are the DNS servers of MNOs to users' queries?

iv. what effect does time of the day has on variables tested?

To find answers to the aforementioned research questions, mobile performance QoS data were collected from several Android Smartphones (for a period of 3 months) using a mobile broadband performance test application (MBPerf) solely developed for this purpose. The detailed development of MBPerf is presented in another paper submitted to [7] . Two cities of Nigeria (Akure and Ibadan) make up the coverage area of the performance study, as these were the volunteers' major residences and the authors' closest reach. Combining the test results in the region gave insightful picture of the performance of each MNO. The approach used and the result from the study could serve as a template that could be adapted for other regions or Nigeria as a whole. This research is a pilot test to investigate the possibility of independent monitoring of mobile broadband performance in Nigeria. Development of the test application is another research focus which this paper does not address; this paper presents the results obtained from the mobile broadband performance test application measurements.

The paper is organised as follows. Section 2 discusses mobile broadband performance and how it relates to development, it also give background information on the state of mobile broadband connectivity in Nigeria. Highlights of mobile broadband measurement approach in comparison to other methods is also presented. Thereafter, there is a discussion on the system architecture and the four (4) QoS metrics featured in our study. Section 3 presents the methodology while section 4 presents and discuss the results. Critical evaluation of the research was presented in section 5 while section 6 presents suggestions to better Internet services in Nigeria. Section 7 concludes the paper.

\section{BAKGROUND}

Mobile broadband performance monitoring helps in guiding the regulator of the telecoms sector in a country in its policy formulation. In addition, when customers are informed on how their Internet services measure up, they are placed in an equitable position to make the right choices for their needs in terms of choosing the fastest and most reliable network and also weighing adequately their needs and budget to make appropriate cost and performance decisions. Furthermore, mobile broadband performance monitoring can be used to promote effective competition amongst MNOs and also hold them accountable for their headline (advertised) speed claims, which in turn will propel them to invest more in their infrastructure to increase capacity and improve customer experiences. 


\subsection{Mobile broadband Performance Analysis in Nigeria}

Mobile broadband networks play an increasingly important role in our society, hence the need for independent and unbiased assessment of their robustness and performance. A promising source of such information is active end-to-end performance measurements [8]. With the current broadband penetration of approximately $30.9 \%$, Nigeria has met her National Broadband Plan target of reaching by the end of 2017, a five-fold increase in broadband penetration over the 2012 penetration rate of between 4-6\% [9]. Yet the International Telecommunications Union (ITU) put the fixed broadband penetration rate at $0.01 \%$. This is an indicator that a large percentage of broadband access is via mobile broadband. Nigeria Communication Commission (NCC) report of Q3, 2017 puts the total active subscriptions of mobile broadband at over 93 million, which is a $1.22 \%$ growth over Q2's over 92 million. Measuring the performance of mobile broadband networks typically entails collecting test samples in order to put them in the right perspective (by sorting and analysing) to be able to give valuable and understandable information about network quality. This has been a continuous activity strictly pursued by all stakeholders, including researchers in the telecommunication industry of the developed countries, such as US, UK, South Africa, Canada, New Zealand etc. Unfortunately in Nigeria, despite the rise in activities in her broadband space and numerous advantages associated with performance monitoring to all stakeholders, little is known about a customercentric mobile broadband performance measurements. The lack of regular researches on mobile broadband QoS in particular must be addressed. Toward this aim, we provide a host and a crowdsourced based study of mobile broadband in two cities of Nigeria.

\subsection{QoS Metrics}

Common metrics that have been employed for measuring broadband performance include upload and download speed, latency, jitter and packet loss [10-13].

Packet Loss: the number of packets in a traffic flow that fails to reach its destination. Packet loss, usually expressed as a percentage can be measured by sending an echo request consisting of small User Datagram Protocol (UDP) packets between a QoS client and the measurement server and then wait for a reply. Packet(s) not received back before the measurement times-out is/are treated as lost.

DNS look-up: A fast Domain Name Service (DNS) is just as important as fast content. DNS is a crucial Internet service that allows domain names such as www.google.com which are human understandable, to be resolved into IP address that the computer can understand.

Latency (delay): Latency is the time it takes a packet to get to its destination when sent from a source measured in milliseconds (ms), it indicates how responsive a network is. Latency affects real time applications such as Voice over Internet Protocol (VoIP) and gaming [6].

\section{Data transfer (upload and download) speed:}

The metric has emerged as the single most commonly cited metric of interest for characterizing the quality of broadband offerings [10]. Data transfer speed (either upload or download) is a measure of the capacity of a user's broadband connection in Mbps, as it indicates the user ability to GET (retrieve) or POST (send) data to the Internet more quickly.

Jitter: This is the variability of latency overtime from point to point and it is generally caused by congestion in an IP network.

\subsection{Broadband Performance Monitoring}

There is no wrong or right method for measuring performance, as each approach has its advantages and drawbacks and various methods are implemented based on resource availability, type of access: wired or mobile (wireless), etc. The most important factor is for the measurement approach to produce a rich data set which when aggregated will reflect the true nature of broadband performance. There are some other related works on performance monitoring and measurements carried out by independent researchers and organisations a summary of these is in Table 1.

A team of Researchers in the RobustNet Research Group at the University of Michigan in 2009, led by Associate Professor Morley Z. Mao, developed MobiPerf, a mobile application and handy network tool to collect anonymous network measurement information directly from end users. Using MobiPerf allows a user to have a good knowledge of his Smartphone's network properties, such as local/ global/gateway IP addresses, cell ID, GPS (latitude and longitude), upload/download bandwidth, signal 
strength, DNS lookup latency, PING latencies, and many more [14].

Chetty et al [6] in 2013 conducted a systematic study of fixed and mobile performance in South Africa using a measurement software (MySpeedTest and Bismark) implemented on mobile phones and home routers respectively. Chetty's performance study alongside the open technical platform embodies an archetypal method of monitoring broadband performance in developing countries [6]. Three QoS performance metrics were considered in the research study: upload and download throughput and latency of connections. They were able to prove that consumers in South Africa experience broadband speeds that are less than what they pay for and this also contrast what is obtainable in the UK and US where ISPs generally deliver advertised speeds.

Similar research was carried out in the UK. Following the conclusion of the $4 \mathrm{G}$ spectrum auction in the UK in 2013, the Office of Communication (Ofcom), UK conducted a research into the performance of the retail 3G and 4G services offered by the UK's four (4) national MNOs namely: EE, O2, Three and Vodafone [15]. From the analysis carried out, Ofcom concluded that $4 \mathrm{G}$ download speeds by all operators were significantly faster than the $3 G$ download speeds. Furthermore, Faggiani et al. [17], in 2014, developed Portolan, a general purpose tool and a crowdsourcedbased system that uses Smartphones as its mobile measuring elements. Portolan's active measurements include traceroute, round trip time and maximum throughput and its passive measurement is Received Signal Strength. Portolan since its launch has been used to build signal coverage maps and also to produce graphs of the Internet at the autonomous system level [16].

The host and crowdsourced based approach was chosen for this study because its cost efficient, robust and has wider coverage across various networks. The approach is independent of the telecommunication regulator and the mobile network operators, it is primarily dependent on customers (volunteer), thereby allowing performance data collection in unbiased and realistic scenarios, so as to get a quantitatively correct impression of the service usage experience from a typical user's point of view.

\section{METHODOLOGY}

The system architecture incorporates all the functioning parts of the performance system and it is presented in Figure 1. It is divided into 3 basic parts: client front-end, communication links and server backend. The client front-end module of MBPerf's system consists of an Android Smartphone and the QoS application (MBPerf) installed on it. The client frontend was primarily employed for performance metrics' measurements (data collection) and logging of the performance results to the online database hosted on a web server with the Uniform Resource Locator (URL) - www.mbperf.com.ng.

Table 1: Comparison of Various Techniques used in Broadband Performance Monitoring

\begin{tabular}{|c|c|c|}
\hline Method & Advantages & Limitations \\
\hline Hardware & $\begin{array}{l}\text { - Requires only a small amount of } \\
\text { user's data quota } \\
\text { - Offer continuous measurements and } \\
\text { capable of producing more accurate } \\
\text { results. } \\
\text { - Little intervention is required from } \\
\text { user after initial installation }\end{array}$ & $\begin{array}{l}\text { - Fairly expensive as it requires upfront costs to } \\
\text { deploy and maintain measurement routers. } \\
\text { - Useful for measuring only the performance of } \\
\text { wired connections. }\end{array}$ \\
\hline $\begin{array}{l}\text { Application } \\
\text { (software) }\end{array}$ & $\begin{array}{l}\text { - Many data points can be collected } \\
\text { from a large number of users with } \\
\text { little additional effort. } \\
\text { - Very cost effective }\end{array}$ & $\begin{array}{l}\text { - Performance results can be affected by users' } \\
\text { biasness, capabilities and configuration of users' } \\
\text { devices (e.g., virus infected phones). } \\
\text { - Useful for measuring only the performance of } \\
\text { mobile or wireless broadband connections. }\end{array}$ \\
\hline Crowdsourced & $\begin{array}{l}\text { - Capable of providing large scale user } \\
\text { base required to make the } \\
\text { performance effort robust } \\
\text { - Monitoring activities can be } \\
\text { parallelized and completed in time. } \\
\text { - Incurs minimal cost. }\end{array}$ & $\begin{array}{l}\text { - Includes human in the control loop. This gives } \\
\text { room for bias and introduction of errors. } \\
\text { - Devices can be turned off according to an } \\
\text { uncontrolled pattern. }\end{array}$ \\
\hline
\end{tabular}


The client front-end communicated with the server back-end via the MNOs' GMSC (for GPRS and EDGE networks) or GGSN (for UMTS networks) and the Internet, which is a Wide Area Network (WAN) based on TCP/IP. The server back-end module was implemented using a web server provided by a hosting company. The server back-end system processed all requests emanating from the client front-end and it served as the target test node for the performance measurements, provided the required storage (data repository) for all the collected data and hosted the data reporting website with the domain name www.mbperf.com.ng.
The web server runs a Windows Operating System with MySQL as the database technology. Data was made available to the administrator's browser via a reporting interface implemented with Microsoft's ASP.NET framework.

\subsection{Data Collection: Deployment and Test Tool (MBPerf)}

This research seek to evaluate the performance of EDGE (2G family), UMTS and HSPA (3G family) networks as delivered to 100 Android Smartphones in different areas of Akure and Ibadan where the 4 target MNOs have adequate presence and provide network services.

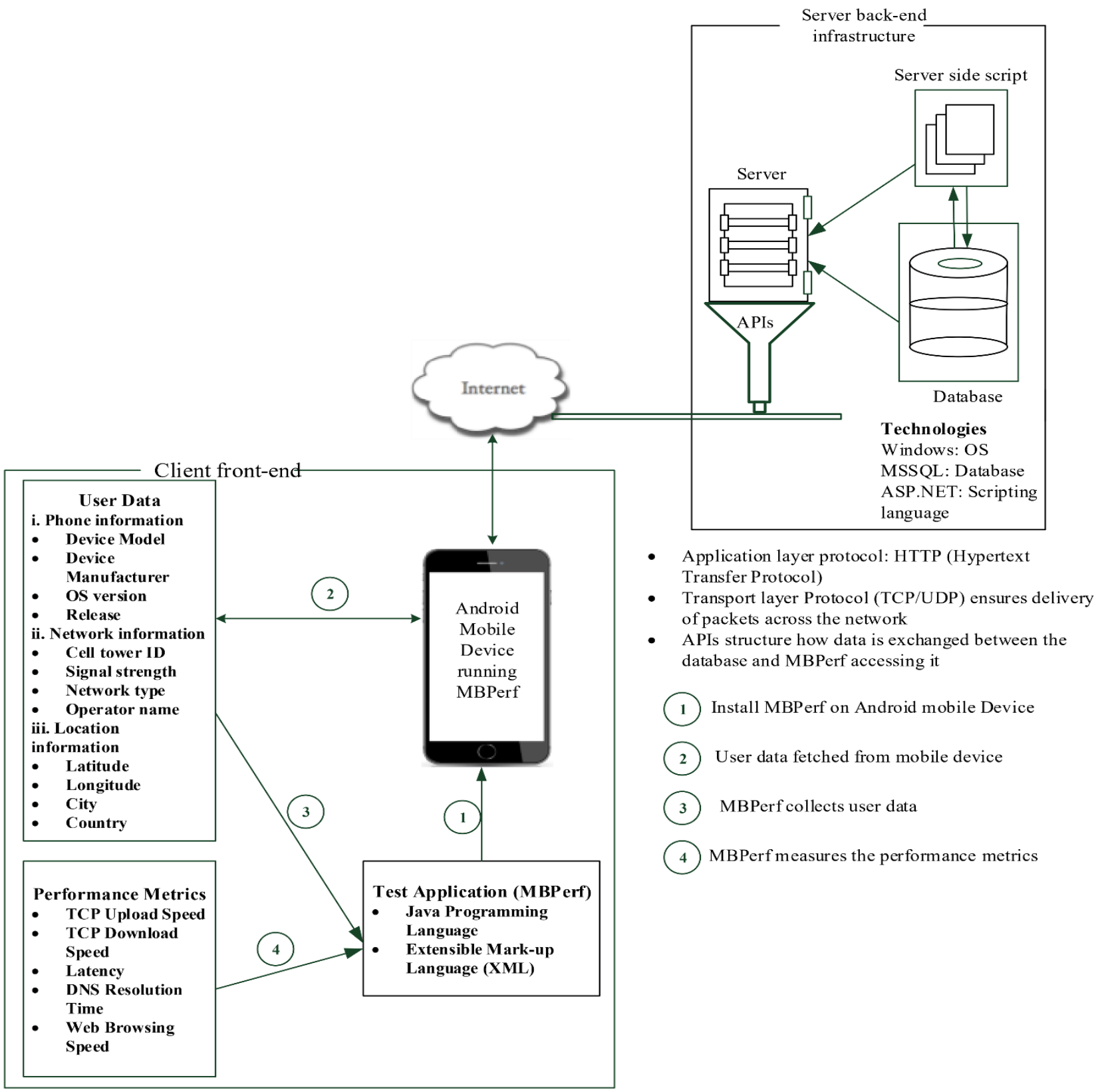

Figure 1: MBPerf System Architecture 
$2 \mathrm{G}$ and $3 \mathrm{G}$ are the predominant types of access network technologies offered by the MNOs and the mobile phones of volunteers. This is because $4 \mathrm{G}$ is currently being rolled out at selected areas of major cities only. Data were collected between January and March, 2018 on mobile connections using the crowdsourced and host based approach. This approach involved developing a QoS application called Mobile Broadband Performance (MBPerf) which was installed on volunteers' Android Smartphones to measure 4 selected mobile broadband QoS metrics download and upload speeds, latency and DNS lookup; including some basic network properties such as network carrier name, network type, cell ID, Location Area Code (LAC), Received Signal Strength (RSS) and the build information of the user's Smartphone.

MBPerf's source code was written in Java programming language while its User Interfaces were designed using XML. All volunteers that partook in the study were recruited via Whatsapp (a social medium platform) and one-on-one campaigns. During the oneon-one campaigns, volunteers recruited included students and staff of The Federal University of Technology, Akure (FUTA), friends and family. Mobile customers who agreed to become volunteers were asked to download MBPerf's apk file from www.mbperf.com.ng. The QoS application (MBPerf) was also deployed unto some volunteers' devices via Xender (a file sharing application). In addition, MBPerf was sent as attachment to email of volunteers who preferred this approach. Once the .apk file has been saved on the user's device, he/she then follows the specified instruction for installation.

Major bottlenecks introduced by the networks were measured because tests were conducted toward an international server. MBPerf ran tests and collected the required metadata from volunteers' Smartphones as a background service (which means users' do not have to worry about initiating tests themselves). The app takes measurements hourly throughout the day. This high frequency testing schedule was allowed so as to achieve robust data set even though users' data consumption increase with frequency of use. Volunteers (users) were provided with the numerical summaries of their mobile connections' performances on their mobile phones. The Android platform was used to carry-out performance measurements to ensure a uniform Operating System (OS) for all volunteers, furthermore, the iOS is widespread, popular and flexible. The speeds (download and upload) and latency measurement conducted toward an international server help reflect bottlenecks that users experience along a wide area path. In addition, most contents users assess are hosted on international servers, therefore conducting tests toward an international facility will allow measurements in more realistic scenarios.

\section{RESULT AND DISCUSSION}

This section presents the comparisons of the average download and upload speed, latency and DNS lookup obtained across the two cities by each of the MNOs on $2 \mathrm{G}$ and 3G. In addition, the average values estimated for each QoS metric were compared with the industry standard values. The results of the time analysis carried out on each of the QoS metric revealed the performance pattern of each of considered MNOs' throughout the day. Though highest numbers of upload and download speeds or the lowest numbers for latency and DNS lookup does not necessarily reflect the best service offered by an MNO or network technology, as other factors can affect performance too. In addition, the measured performance in Q1, 2018 may not reflect the current or future performance of the MNOs and locations tested.

\subsection{Coverage Mapping of the Study Area}

The coverage map of all the areas covered by MBPerf deployments in the cities of Akure and Ibadan are shown in Figures 2 and 3 respectively. The maps were developed using ArcGIS software developed by ESRI. The map also has data points that reveal different tests sites where performance results were collected. The latitude and longitude as well as the signal strength values obtained per measurement for all users were used to map the areas covered by MBPerf deployment in the two cities. In summary, the data points on the coverage maps when compared with the signal strength power levels shown on the legends which suggest that the 4 MNOs' $^{\prime}$ have considerably good coverage in both cities.

\subsection{HTTP Download and Upload Speed Analysis}

The MNO with the fastest average 3G download speed recorded across all of the sample sites in Akure was MNO-A, at $511 \mathrm{Kbps}$. This was not the case in Ibadan, where MNO-D (475.7 Kbps) recorded the fastest average download speed. MNO-B on its part had the lowest average 3G download speed at $450.2 \mathrm{Kbps}$ while MNO-C and MNO-D recorded the average $3 \mathrm{G}$ download speeds: $495.4 \mathrm{Kbps}$ and $485.5 \mathrm{Kbps}$ 
respectively as shown in Figure 4 . For MNO-A, the average $3 \mathrm{G}$ download speed in Akure was almost twice that offered in Ibadan. It is to be noted that no statistical significant difference exists between the average download speeds offered by the 4 MNOs in Akure.

Comparing the average download performance in Ibadan, MNO-D had the fastest average speed of 475.7 Kbps, followed by MNO-B, which recorded 437.7 Kbps. The average speed of MNO-D and MNO-B are significantly faster than MNO-A, but no significant difference exists between MNO-C and MNO-A's average download speeds. Figure 4 also reveals that all MNOs had higher $3 \mathrm{G}$ average download speeds in Akure than in Ibadan. This is similar to the overall result where the overall $3 \mathrm{G}$ download speed in Akure is faster than in Ibadan.

In Figure 5, MNO-C and MNO-A had 3G upload speeds that were above overall average in Akure, with MNOC's $3 \mathrm{G}$ upload speeds being the highest at $401.1 \mathrm{Kbps}$. MNO-B and MNO-D were both below the overall average 3G upload speed. There exist no statistical significant difference between MNO-C (401.1 Kbps) and MNO-A's average upload speed (396.8 Kbps). However, in Ibadan only MNO-C performed above the overall average upload speed at $360.3 \mathrm{Kbps}$ making it again the fastest amongst the three MNOs.

Volunteers in the MBPerf deployment were asked via a Whatsapp opinion pool about their perceptions of speed for common Internet activities. Interestingly, about $82 \%$ of the respondents replied that speeds were acceptable for services such as news, Facebook, email, and music download. However, more than half of the users rated high bandwidth services such as video streaming, watching videos and photo upload on the average, as they are not satisfied with the service offered. Recall that bandwidth intensive services require a consistent data rate of between $0.5 \mathrm{Mbps}$ and 3.2 Mbps while bandwidth non intensive activities require a bandwidth of between $0.1 \mathrm{Mbps}$ and 0.3 Mbps [17]

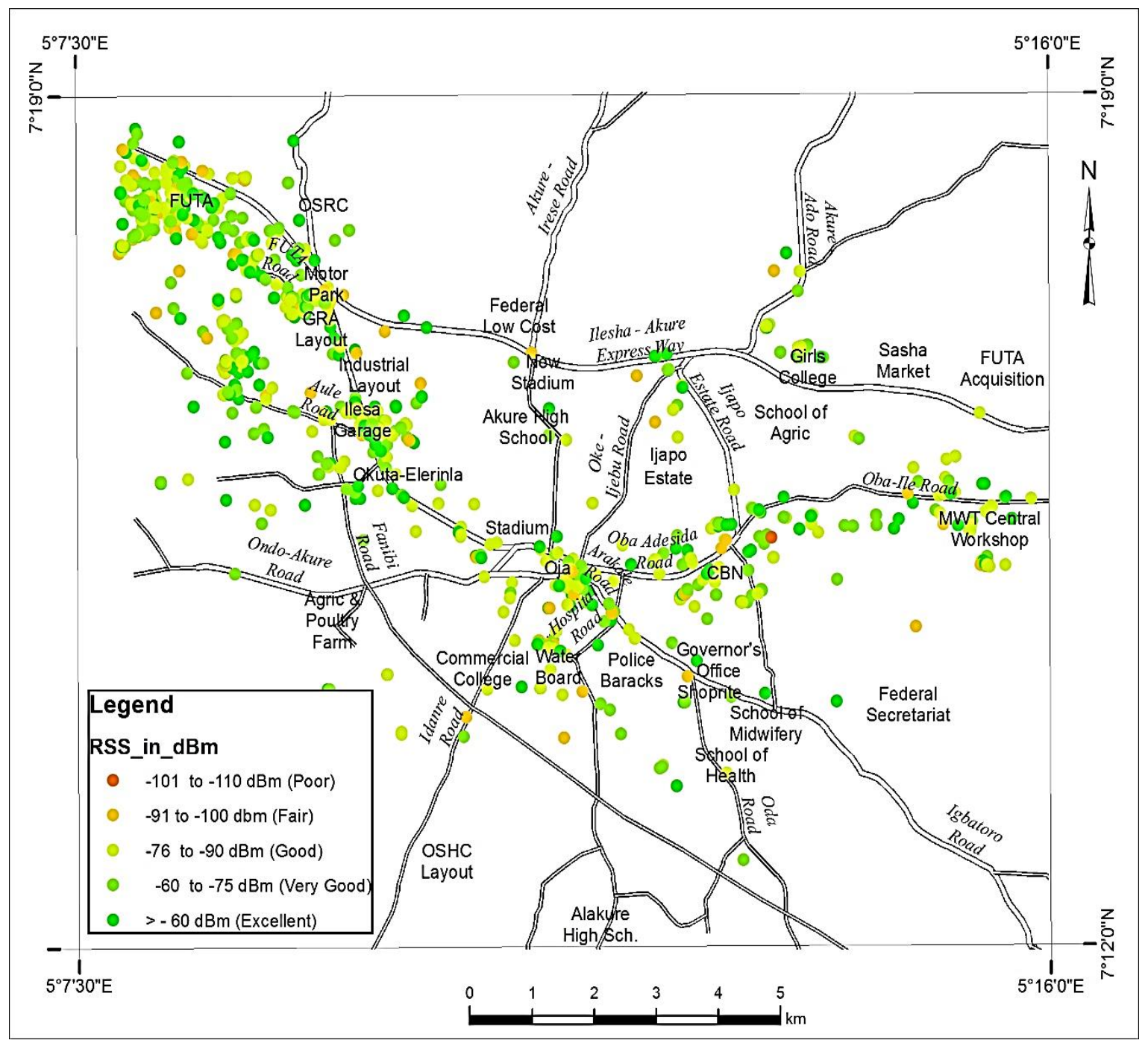

Figure 2: MBPerf Deployment in Akure Metropolis 


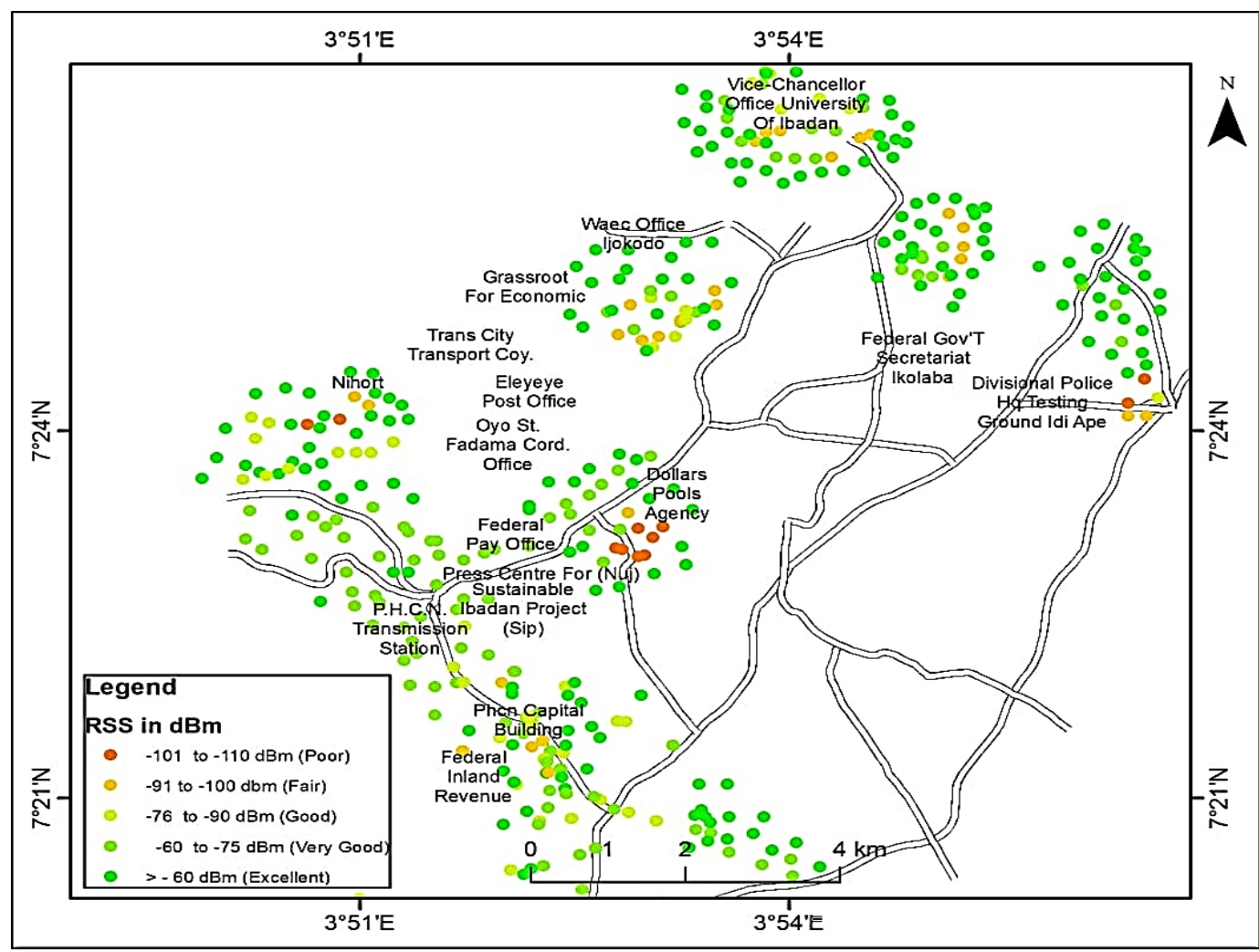

Figure 3: MBPerf Deployment in Ibadan Metropolis

\subsection{Latency Analysis}

Figure 6 shows a comparison between the average latency results obtained for all the MNOs in Akure and Ibadan. Amongst the MNOs, the data aggregated for Akure shows that MNO-C ranked the most responsive network with an average latency of $148.12 \mathrm{~ms}$, followed by MNO-B, at $167.83 \mathrm{~ms}$. MNO-A had the highest average latency of $283.91 \mathrm{~ms}$. The latency performance achieved by MNO-C is significantly better than all the other 3 MNOs. On the hand, in Ibadan; MNO-D had the least average latency of $234 \mathrm{~ms}$, signifying the most responsive network. MNO-A again had the most latency of $293.2 \mathrm{~ms}$. It was also inferred that Latency on paths from Akure to Arizona (the location of the measurement server) is less than from Ibadan to Arizona while the download and upload speeds measured in Akure were higher than in Ibadan. This is likely because TCP speed is inversely proportional to the round-trip latency between two communicating hosts.

Figure 7 shows that less people use the network (i.e. frequency of connection to the various networks is reduced) between $12 \mathrm{am}$ in the night and $6 \mathrm{am}$ in the morning which translates to a reduced latency at this period and less congestion. A strong and positive correlation $(r=+0.66)$ exist between the number of users on all networks and the average latencies.
Therefore, congestion and reduced performance are expected in the day time and late in the evening.

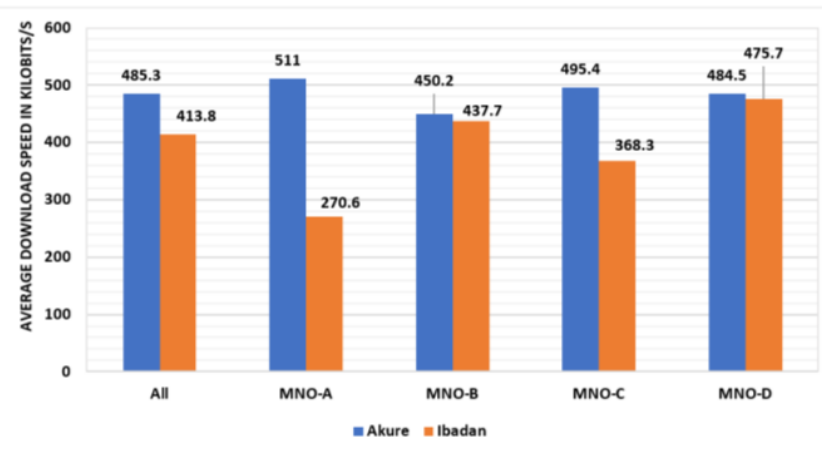

Figure 4: Average 3G HTTP Download Speeds, by MNO in Akure and Ibadan (higher is better): Q1, 2018

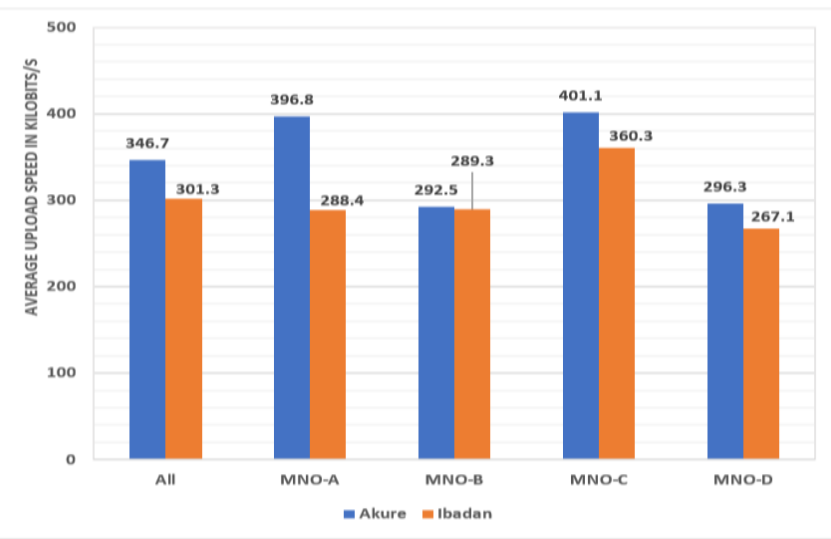

Figure 5: Average $3 G$ HTTP Upload Speeds, split by MNO across Locations (higher is better): Q1, 2018 


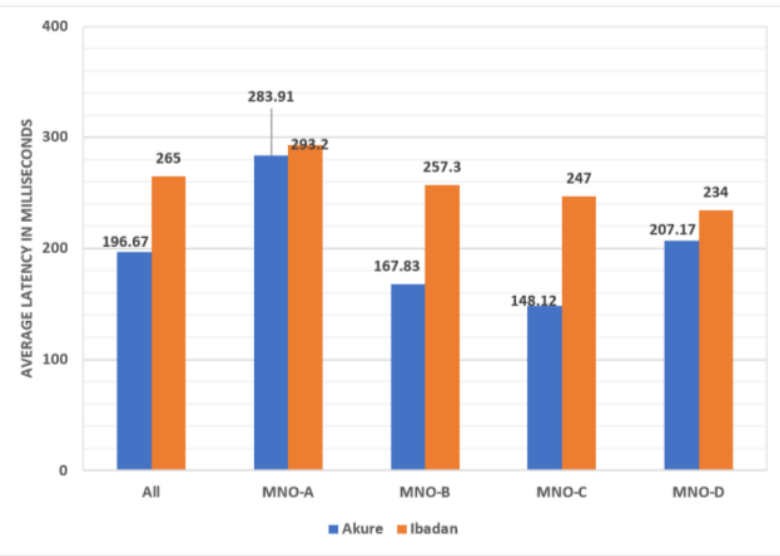

Figure 6: Average 3G Latency, by MNO (lower is better): Q1, 2018

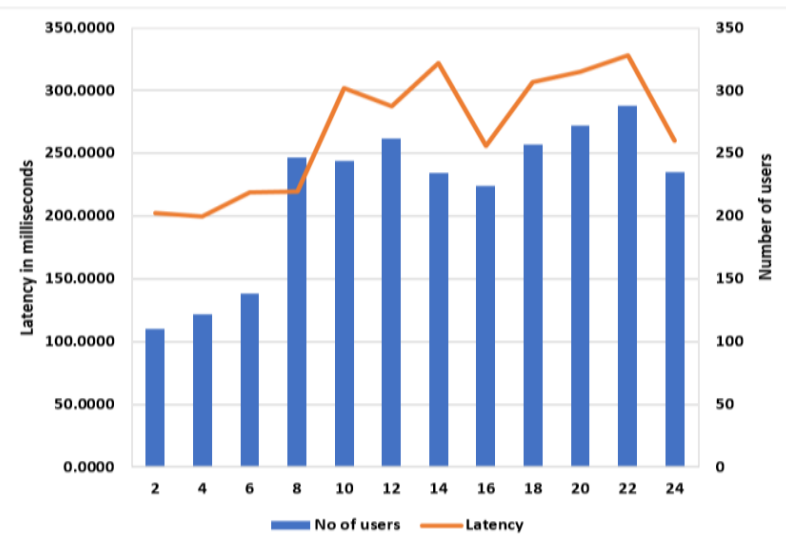

Figure 7: The Frequency of Network Use per Hour and Latency, by Local Time of Day

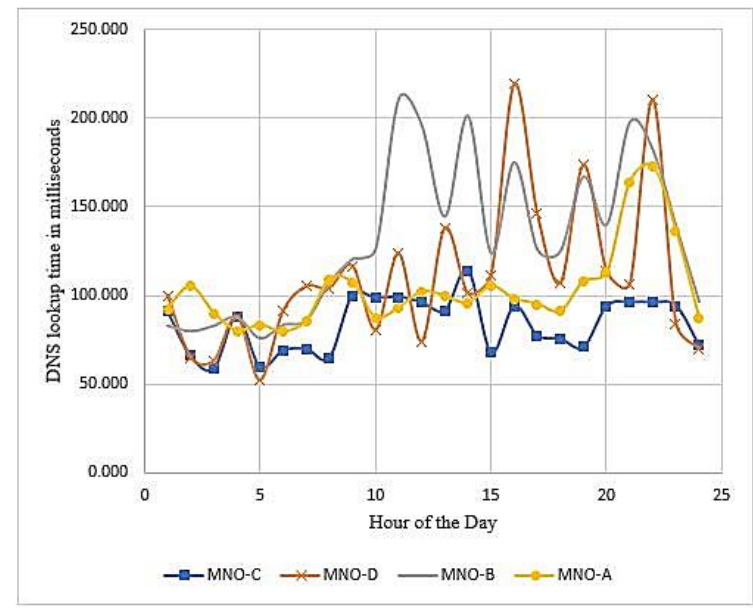

Figure 8: 3G DNS resolution time in Akure, split by hour of the day and MNO

\subsection{DNS Lookup Analysis}

Table 2 shows the comparison between the DNS resolution time throughout a 24 -hour profile and during peak hours. For the purpose of this analysis, peak hour is assumed to be between $7 \mathrm{pm}$ (19 hours) and $11 \mathrm{pm}$ (23 hours) inclusive. DNS resolution tends to be higher for MNO-B than for MNO-C, MNO-D and MNO-A, and there exist a significant difference between MNO-B's peak DNS performance and the overall 24-hour performance. The other 3 MNOs apart from MNO-B demonstrated a lower amount of time taken (Table 2) to resolve DNS queries, with MNO-C almost completely unaffected by peak hour traffic, as was the case with its latency values which were negligibly variable and relatively lower than other latency characteristics exhibited by the rest MNOs during the study period. Figure 8 shows 3G DNS resolution time in Akure, split by hour of the day and MNO.

Taking a closer look at the 24-hour average DNS results stated in Table 2 . The values are slightly above $100 \mathrm{~ms}$ as obtained in Akure across the 4 MNOs, it will be seen that they are considerably lower (better) than latency measurements (shown in Figure 6). This is suggestive of the fact that the MNOs' DNS servers are in general operating effectively and not adding significant delay to end users' queries because according to the submission made in 2012 by [18]; "in theory, a good DNS deployment should provide DNS resolution time and failure rates better than or equal to the latency and packet loss figures." Probably it is because DNS servers are hosted inside the Internet Service Providers' (ISPs') networks and therefore the DNS packets often times transverse only the ISPs' networks.

\subsection{Effect of Time of the Day on Metrics 4.5.1 Effect of Time of the Day on Download and Upload Speed}

Studies in the past [13] and [19] revealed that users' perceive inconsistent performance because the network can become more congested at certain times of the day (known as peak hours). Figure 9 shows the download speed metric plotted against hours of the day. MNO-C users experience an average $46 \%$ drop in performance between early morning (around $3 \mathrm{am}$ ) and evening time (around $9 \mathrm{pm}$ ) when users are most likely back from work.

Table 2: $3 G$ peak and 24-hour DNS resolution time in Akure, split by MNO

\begin{tabular}{cccccc}
\hline Period/MNO & MNO-C & MNO-D & MNO-B & MNO-A & ALL \\
\hline Peak & $94 \mathrm{~ms}$ & $113.5 \mathrm{~ms}$ & $167 \mathrm{~ms}$ & $113 \mathrm{~ms}$ & $113.3 \mathrm{~ms}$ \\
\hline 24-hour & $92 \mathrm{~ms}$ & $108 \mathrm{~ms}$ & $121 \mathrm{~ms}$ & $101 \mathrm{~ms}$ & $105 \mathrm{~ms}$ \\
\hline
\end{tabular}


MNO-D users witnessed a maximum download peak time at noon with a drop in performance from $12 \mathrm{pm}$ onward till late at night (11 pm). For MNO-B, peformance drop (difference between the peak download speed and the worst download speed) is about $53 \%$ while for MNO-A, it is about $69 \%$. This percentages show that there exists a significant variation in performance between the early morning and evening service quality. The graph also shows, performance variability increases for all MNOs especially during the day between 8am in the morning and $5 \mathrm{pm}$ in the evening. The biggest difference between peak and worst performance is $69 \%$ variation.

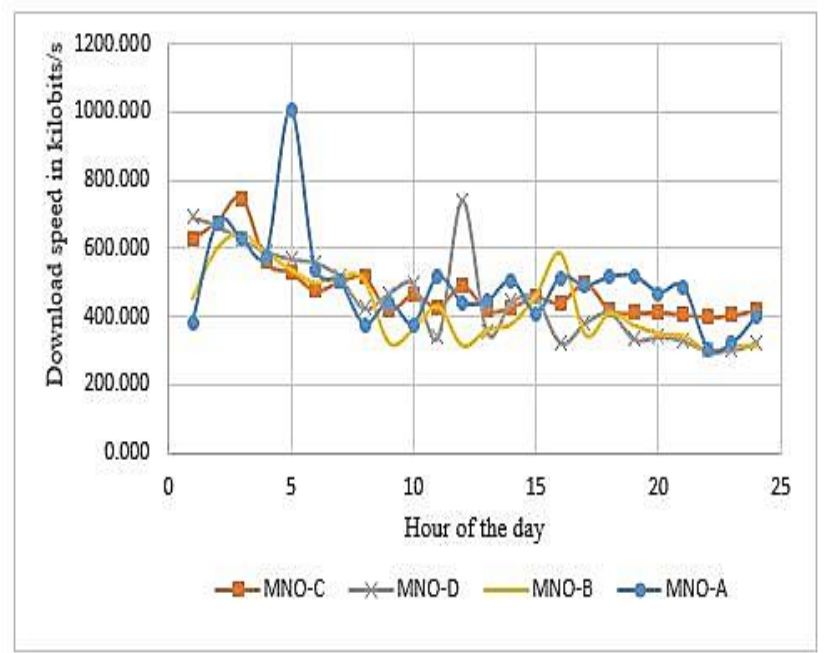

Figure 9: Effect of Time of the Day on Download Speed Performance

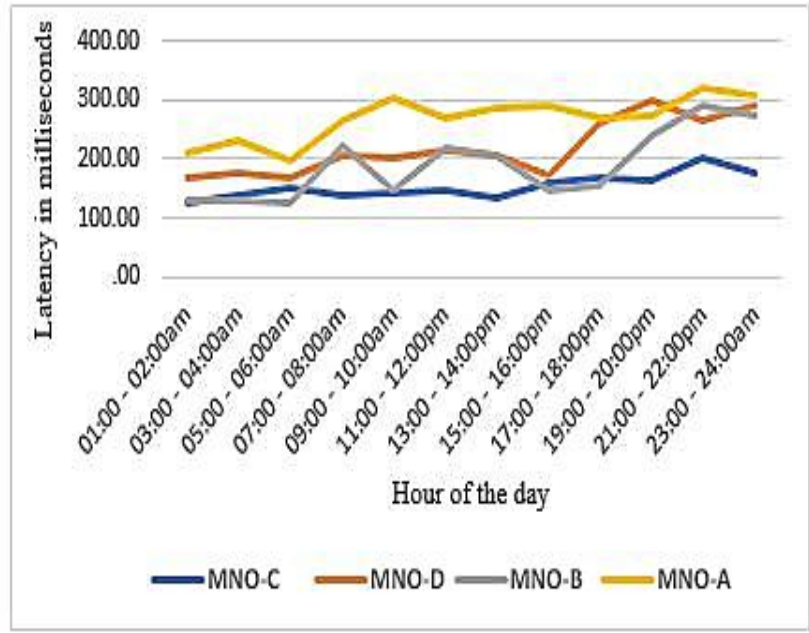

Figure 10: Median Latency Values Plotted against the Local Time of the Day from Different Test locations in Akure to the Measurement Server

In summary, there is significant decrease in download performance during the evening hours (between $7 \mathrm{pm}$ and $12 \mathrm{am}$ ) as well as high performance variation of
Quality of Service during the day. However, performance greatly improves between 1am and 5am.

\subsubsection{Effect of Time of the Day on Latency}

Latency measurements for MNO-B shows a very unstable performance throughout the day and only MNO-C had more stability. Latencies were seen to be consistent throughout the early hours of the morning for all the operators as shown in Figure 10. Even though latencies towards the measurement server are quite high (above $200 \mathrm{~ms}$ ) especially for MNO-D, MNO$A$ and $M N O-B$; there exists a significant improvement in performance achieved (about $60 \%$ ) between the early hours of the morning (between 1 am and $6 \mathrm{am}$ ) and the evening till late in the night (between $4 \mathrm{pm}$ and $10 \mathrm{pm}$ ). The biggest difference between best (for MNO-C) and worst performance (for MNO-A) is over $60 \%$.

\subsection{Comparison between $2 \mathrm{G}$ and $3 \mathrm{G}$ networks}

Taking a closer look at the results shown in Figures 12 and 13 , it is seen that across all networks in the two cities tested, the overall average $3 \mathrm{G}$ download speed was about 3 times faster than the average $2 \mathrm{G}$ upload speed. This is expected; uploading or downloading content such as photographs or videos from the Internet using a $3 \mathrm{G}$ connection would be faster than doing so over a $2 \mathrm{G}$ connection. Primarily because in $2 \mathrm{G}$, the usage of spectrum is insufficient and also the number of bits of information packed into transmitted symbols are less, as low order modulation schemes (like GMSK) are used. Unlike 3G, which uses mutually orthogonal spreading codes (OFDM techniques), that allows better usage of the spectrum and higher modulation techniques like QPSK, 16QAM and 64QAM that increase the number of bits in each transmitted symbol [20].

In Figure 11, a comparison was made between the average latency results achieved on $3 G$ and $2 \mathrm{G}$ networks. The graph show delays experienced by the $3 \mathrm{G}$ users toward the measurement server (in Arizona, USA) were about 3 times lower (faster and better) than those obtained on 2G; most likely because 3G being a higher access technology than $2 \mathrm{G}$ has as one of its advantages the capacity to offer a reduced latency toward destinations [21]. This difference will be particularly noticeable when a $2 \mathrm{G}$ user and a $3 \mathrm{G}$ user both engage in VoIP calls or video call. 


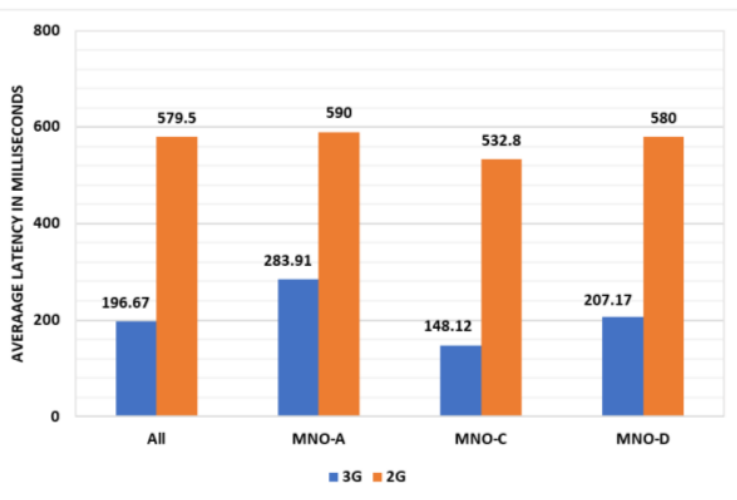

Figure 11: Average $3 G$ and $2 G$ Latency, by MNO (lower is better): Q1, 2018

\subsection{Matching Metrics with Industry Standard 4.7.1 Matching Download Speed Performance with Industry Benchmark}

The box and whisker plot in Figure 12 shows the distribution of $3 \mathrm{G}$ download speed values obtained in Akure. The industry benchmark is given by [22] as 500 -5000 kilobits per second. These results show that in most cases, users in the study do not achieve the industry set values or it can be said that MNOs did not meet benchmark (as all average values are below the lower limit of the benchmark). However, occasionally, users get download speeds that exceed the lower limit of the benchmark as shown by the upper whiskers. Performance for MNO-B and especially MNO-A is highly variable, where users sometimes experience relatively significant high download speeds, as shown by the upper whiskers. Notice also that the performance of MNO-C and MNO-D are relatively more consistent than that of MNO-B and MNO-A. All the 4 MNOs examined fall short of matching the industry benchmark as indicated by the red bar line.

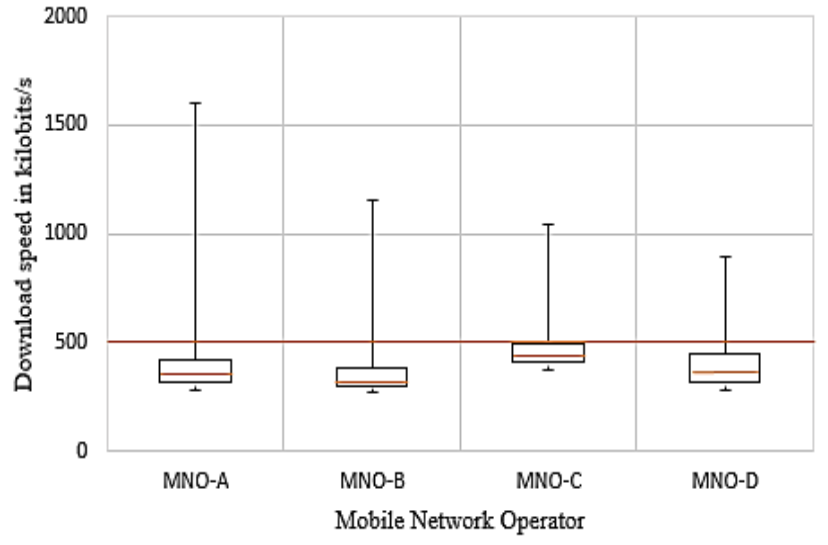

Figure 12: Download Speed for each MNO compared with Industry Benchmark

Figure 13 shows the distribution of $2 \mathrm{G}$ download speed values obtained at different sites in Akure. These results show that users achieve the industry set values which ranges between 100 and 400 kilobits per second [22], as the average values obtained for each MNO fall within these benchmark values. All 3 MNOs examined met the industry benchmark of $2 \mathrm{G}$ speed as indicated by the red bar lines and difference in performance between the 3 MNOs is not significant.

Upon further investigation, there were few cases where users were receiving download speeds that were significantly less than the upper limit of the benchmarks.

Note that the benchmark values lie between 500 and 5000 kilobits per second. From these Figures, it can be seen that the download speeds provided by MNOs did not meet the industry benchmarks. It is unfortunate that customers only records below 500 Kbps most times.

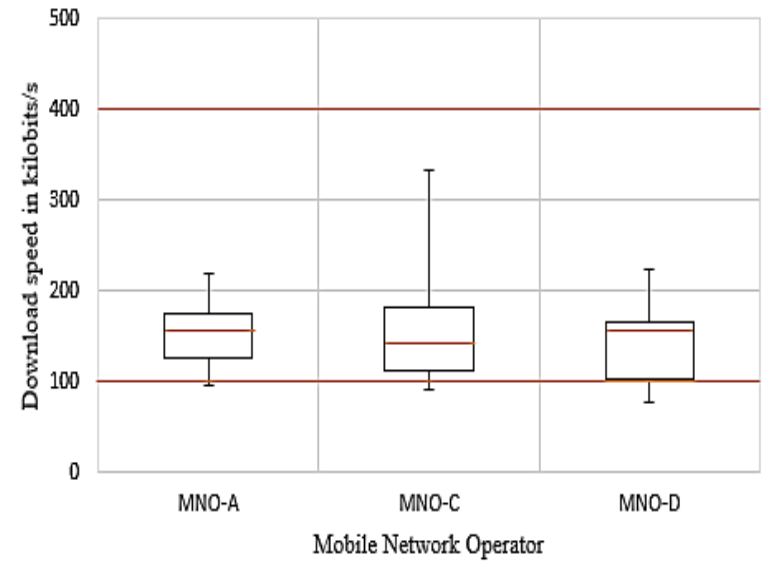

Figure 13: $2 G$ Download Speed for each MNO compared with Industry Benchmark

These results are objective, given that mobile customers in Nigeria complain of slow speeds and limited bandwidth. Still, the magnitude of the performance difference has great contrast with the findings from similar studies in developed countries such as London [15] and South Africa [6] where the estimated average download speeds were 5.9 Mbps and 3.5 Mbps respectively.

\subsubsection{Matching Upload Speed Performance with Industry Benchmark}

MNO-C in the study had the highest average upload speed and distribution of its download speed values was the most consistent, as shown in Figure 14. It is important to note that performance across different MNO-A users is highly variable where users sometimes experience a relatively significant high upload speeds as revealed by its upper whisker. As inferred from the download speed results, none of the MNOs met 
industry standard values which lie between 500 and $5000 \mathrm{Kbps}$ [22] as shown by the red bar line.

For upload speeds measured across different test sites in Ibadan, Figure 15 shows that MNO-C still performed better than the other 3 MNOs. MNO-D users experience the lowest upload speed values. Furthermore, MNO-B, MNO-C and MNO-D users sometimes witness significantly high upload speeds with variable distribution of download speed values than MNO-A users, as revealed by the whiskers. In summary, none of the MNOs (in Akure or Ibadan) met either the industry benchmark values [22] or values estimated in a similar study conducted in UK [15] where the overall average upload speed reported was 1.6 Mbps.

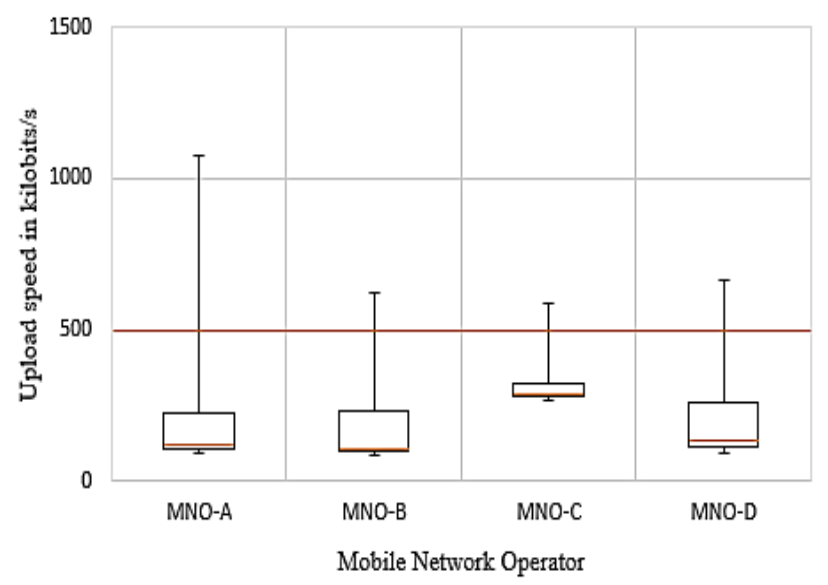

Figure 14: Upload Speed for each MNO in Akure Compared with Industry Benchmark

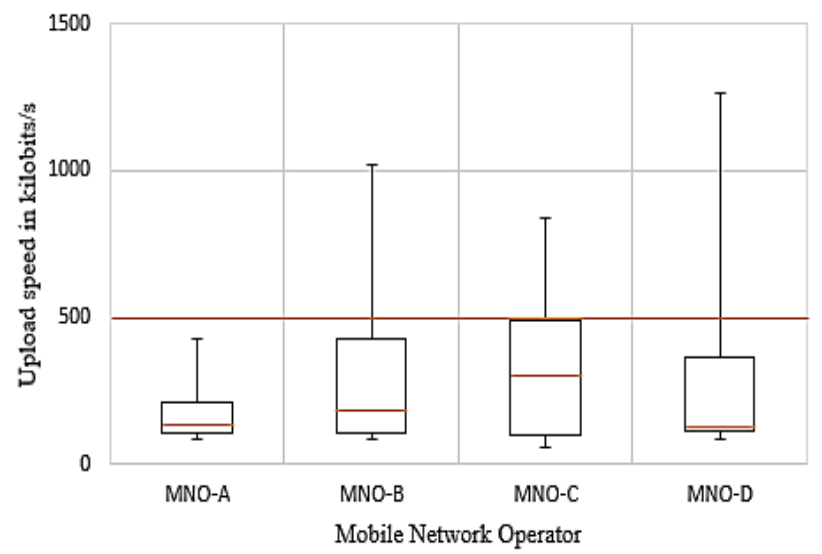

Figure 15: Upload Speed for each MNO in Ibadan compared with Industry Benchmark

\subsubsection{Matching Latency Performance with Industry Benchmark}

Results reveal that latencies are incredibly variable and some MNOs have much more variable latency than others shown in Figures 16 and 17. Notice in Akure and Ibadan that MNO-C had the most consistent distribution of latency values.

The industry standard for 3G latency is given by [22] as $100-500 \mathrm{~ms}$, while for $2 \mathrm{G}$ latency is given as 300 - 1000 ms. Figures 16 show that the median values achieved by each of the MNOs fall within the industry set standard. This was also the case for $2 \mathrm{G}$ latencies in Figure 17. Even though, occasionally, users see latency that exceed the benchmark as shown by the upper whiskers.

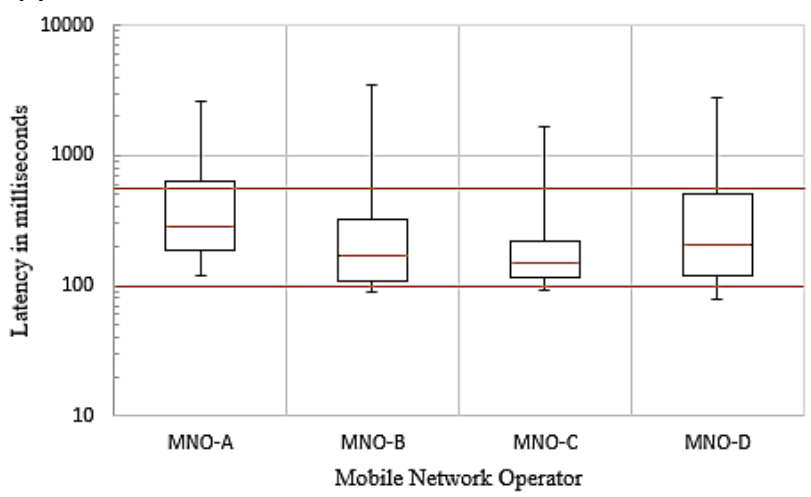

(a)

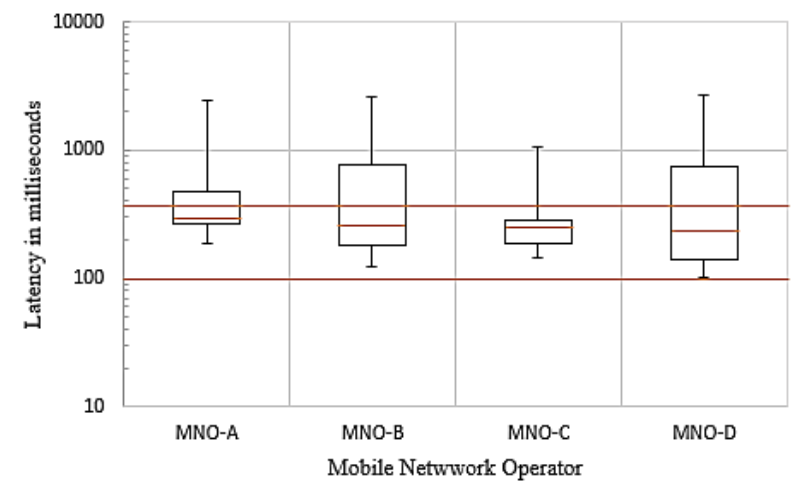

(b)

Figure 16a,b: $3 G$ Latency of MNOs' in Akure and Ibadan respectively compared with Industry Standard

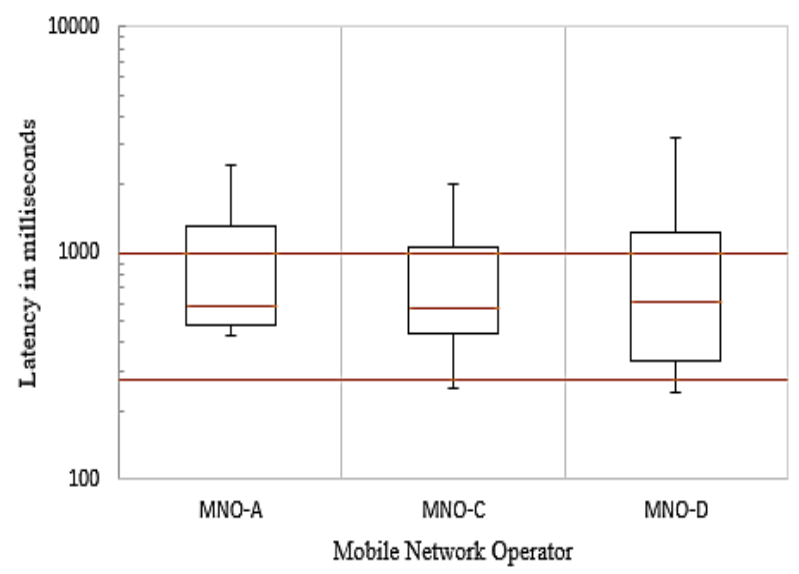

Figure 17: 2G Latency for each MNO compared with Industry Standard 


\section{CRITICAL EVALUATION OF RESEARCH}

Firstly, higher frequency of data collection provides more robust data set about time-varying properties of a customer's Internet connection but also risks exhausting the customer's monthly data subscription. It is against this back drop that all configured tests were carried out hourly on the mobile devices throughout the period of data collection. Secondly, recruiting volunteers required much effort because many customers had privacy concerns even though MBPerf does not collect any personal identifiable information of the volunteers. Some customers equally gave insufficient memory requirement as an excuse for not installing the application at all or uninstalling it after initial download and installation.

Third, volunteers were weary that putting on their device's GPS (location) will quickly run down their battery charge, unfortunately not putting it on regularly prevented MBPerf from logging their location information at certain times. Hence, some measurements were inevitably discarded, since location of users is one of the explanatory variables of the MBPerf dataset. Fourthly, the data collection was seamless and did not disrupt users' Internet access. The measurement cycle takes less than 2 minutes to complete and was run as a background service.

Finally, efforts were made in order to get Key Performance Indicator (KPI) standard values from MNOs or NCC in Nigeria to benchmark some (if not all) of the results obtained. Unfortunately, NCC until the time of writing this paper does not have on its website any minimum QoS standard KPI for data service delivery, which can be used to ascertain operators' performance; even though it has for voice services. It was against this backdrop that benchmarks available for speed and latency in literature [22] were used.

\section{SUGGESTIONS TO BETTER INTERNET SERVICE IN NIGERIA}

The $3 G$ upload and download speed performance offered by the four major MNOs in the first quarter of 2018 presented in this paper, suggests that the MNOs' 3G networks are overburdened and are facing demands that pushes network capacity to its limits, particularly in the densely populated urban areas such as those considered in the research (Akure and Ibadan). This capacity crunch can be attributed to the significant growth in the number of $3 \mathrm{G}$ users as well as in the volume of mobile traffic traversing the MNOs' networks; following the proliferation of Smartphones and new mobile devices, which supports a wide range of applications and services. As such, the following are suggested ways by which the MNOs can increase the capacity of their networks quickly, effectively and economically.

i. Network densification: MNOs can leverage on the recent innovations and developments in densification through addition of more cell sites underneath their macro network layers; as having more sites allow for greater spectrum (frequency) re-use, thereby increasing capacity.

ii. Spectrum availability: MNOs can expand the bandwidth available to them by leveraging on the conditional access to the $3.5 \mathrm{GHz}$ and unlicensed $5 \mathrm{GHz}$ bands.

iii. Network capacity management: With capacity management, the MNOs can influence the behaviour of their networks by configuring QoS rules aimed at controlling the available capacity in order to know what (percentage of) service needs to be limited at certain times and at different areas (locations).

Furthermore, it is believed that this research effort has provided a reliable reference point and architecture that NCC can expand on for the continued study of mobile broadband performance in Nigeria. Hence, it is strongly recommended that NCC should institute continual longitudinal measurement and reporting of mobile broadband performance in the country for the various MNOs by increasing the volunteer base of this research effort and consequently the number of measurements through rigorous recruitment campaign protocols including incentives for volunteers; so that enough data can be pulled quarterly for analysis.

The MNOs should make available detailed and concise information about the various data packs that consumers buy from their outlets. For instance, bandwidth provided should be explained in unequivocal terms and then misleading advertising should be avoided. In addition, MNOs should put into the public space (website) their performance reports. This would provide another avenue for information dissemination to consumers that will help them make informed choices.

A system of ranking needs to be put in place so that MNOs can be ranked regularly based on their relative performances in relation to different QoS parameters. In so doing, healthy competition will be fostered amongst MNOs, efficiency and innovation will be greatly enhanced. The ranking system is an incentive for MNOs to provide better services to their consumers. 


\section{CONCLUSION}

In this research, a QoS application (MBPerf) was developed, which measured four (4) selected performance metrics and stores the Key Performance Indicator (KPI) estimate alongside information relating to a user's network, phone and location on an online database. The analysed data gave insights into performance offered to customers by the MNOs in the two cities that formed the coverage area of the study. The results indicated four major conclusions. Firstly, the MNOs did not meet the industry benchmark on 3G, though $3 \mathrm{G}$ outperforms $2 \mathrm{G}$ services. Secondly, time of the day and congestion are important factors that affect the overall performance that mobile users get. Thirdly, performance that users achieve are generally highly variable (inconsistent and unstable) especially during the day and at peak times (between $7 \mathrm{pm}$ and $11 \mathrm{pm})$, but greatly improves in the early hours of the morning (between $1 \mathrm{am}$ and $6 \mathrm{am}$ ). Finally, the MNOs' DNS resolvers are performing effectively and do not add significant delays to users' queries.

The results outlined in this paper show that there is a distinct need to make available consistent, longitudinal (continual) and open measurements of mobile broadband performance for MNOs in Nigeria, as data emanating from the performance studies can help policymakers make arguments as regards regulatory or financial incentives for MNOs that provide improved services to their customers. Even though the results in our research are specific to just two cities in Nigeria, it is expected that many of the general findings hold true in other cities and it is hoped that this research be extended to other cities and regions of Nigeria in future works.

\section{ACKNOWLEDGEMENT}

The authors would like to appreciate the Tertiary Education Trust Fund (TETFund), Nigeria for supporting with a University based Research Grant.

\section{REFERENCES}

[1] Federal Communications Commission(FCC), "Types of Broadband Connections," Federal Communications Commission, 23 June 2014. [Online]. Available: https://www.fcc.gov/general/typesbroadband-connections. [Accessed 21 February 2019].

[2] J. Junaid, "Network-based Monitoring of Quality of Experience (A Ph.D Dissertation)," Department of Communications Systems,
Blenkinge Institute of Technology, Karlskrona, Sweden, 2015.

[3] Nigerian Communications Commission, "NCC Stakeholder Information," Nigerian Communications Commission, 2018. [Online]. Available: Available: https://www.ncc.gov.ng/stakeholder/statisticsreports/industry-overview\#view-graphstables. [Accessed 20 August 2018].

[4] Ericsson, "Mobility Report: On the Pulse of the Networked Society," Stockholm, Sweden, 2016.

[5] Government of Nigeria, "Nigeria's National Broadband Plan 2013 - 2018," 2013. [Online]. Available:

https://www.researchictafrica.net>national broadband plan 2013-2018.pdf. [Accessed 2 April 2017].

[6] M. Chetty, S. Sundaresan, S. Muckaden, N. Feamster and E. Calandro, "Measuring Broadband Performance in South Africa," in 4th Annual Symposium on Computing for Development (ACM DEV), Cape Town, South Africa, 2013.

[7] A. Akinlabi and F. M. Dahunsi, "Performance Monitoring of Mobile Broadband in a Developing Country," in Submitted to ISTAfrica Conference, May, 2019, Kenya, 2019.

[8] B. Dziugas, E. Ahmed and K. Amund, "Measuring the Reliability of Mobile Broadband Networks," in 2014 Internet Measurement Conference, Vancouver, Canada, 2014.

[9] B. Okunoye, "Broadband Access in Nigeria: Not Broad Enough, Not Qualitative Enough (Policy Brief)," 2018. [Online]. Available: http://nigeriacommunicationsweek.com.ng/br oadband-access-in-nigeria-not-broad-enoughnot-qualitative-enough-report/. [Accessed 2 March 2018].

[10] S. a. L. W. Bauer, "Understanding Broadband Speed Measurements," 2010.

[11] Federal Communications Commission, " Measuring Broadband America July Report: A Report on Consumer Wireline Broadband Performance in the U.S," USA, 2012.

[12] Ofcom, " UK Broadband Performance May 2012: The Performance of Fixed-Line Broadband Delivered to UK Residential Consumers," UK, 2012. 
[13] S. Sundaresan, R. Teixeira, N. Feamster, A. Pescape, S. Crawford and W. De Donato, "Broadband Internet Performance: A View from the Gateway," in ACM SIGCOMM 2011 Conference, Toronto, Ontario, Canada, 2011.

[14] U. Goel, M. P. Wittie, K. C. Claffy and A. Le, "Survey of End to End Mobile Network Measurement Testbeds, Tools and Services," IEEE Communications Surveys and Tutorials, vol. 18, no. 1, pp. 105-123, 2016.

[15] Office of Communication (Ofcom), "Measuring Mobile Broadband Performance in the UK: 4G and 3G Network Performance," United Kingdom, 2014.

[16] A. Faggiani, E. Gregori, L. Lenzini, V. Luconi and A. Vecchio, "Smartphone-based Crowdsourcing of Network Monitoring: Opportunities, Challenges and a Case Study," IEEE Communications Magazine, vol. 52, no. 1 , pp. 106-113, 2014.

[17] L. Ken, "Download Speeds: What do 2G, $3 G$ and 4G Actually Mean to You?," kenstechtips, 2018. [Online]. Available: https://www.kenstechtips/index.php/download -speed-2g-3g-and 4g-actual meaning. [Accessed 17 August 2018].

[18] European Commission, "Quality of Broadbnad Services in the EU," Luxembourg, 2012.

[19] S. Sundaresan, R. Teixeira, N. Feamster, S. Crawford and A. Pescape, "Measuring Home Broadband Performance," Communications of the ACM, vol. 55, no. 11, pp. 100-109, November 2012.

[20] A. R. Mishra, Cellular Technologies for Emerging Markets: 2G, 3G and Beyond, First ed., West Sussex: John Wiley and Sons Ltd, 2010.

[21] I. Grigorik, Primer on Latency and Bandwidth: High Performance Browser Networking, Sebastopol: O'Reilly Media Inc., 2013.

[22] G. Ilya, High Performance Browser Networking: What Every Web Developer should Know about Networking and Web Performance, 1st ed., C. Nash, Ed., Sebastopol: O'Reilly Media, Inc., 2013. 